\title{
Trauma psicosocial y estrés postraumático en las "viudas blancas" por la explosión de un ducto de gasolina en el Municipio de Tlahuelilpan, Hidalgo
}

\author{
Psychosocial stress and post-traumatic stress in the "white widows" \\ due to the exploitation of a gasoline pipeline \\ in the municipality of Tlahuelilpan, Hidalgo
}

\section{Cecilia López-Pozos}

Universidad Autónoma de Tlaxcala, México

Correo: clpozos.proyecto@gmail.com

Resumen: El presente trabajo realiza un análisis de las consecuencias del trauma psicosocial que vivieron "las viudas blancas", mujeres indígenas que perdieron a sus parejas en la explosión de los ductos de Petróleos Mexicanos (Pemex), el 18 de enero de 2019, en el Municipio de Tlahuelilpan, Hidalgo, México. El estudio fue basado teóricamente en el trauma psicosocial y la metodología cualitativa, con un diseño etnográfico, aplicando las técnicas de observación participativa, entrevista en profundidad y diario de campo, realizado con una muestra de diez mujeres, de 30 a 60 años de edad. Los resultados muestran que la alta vulnerabilidad social que padecen las viudas en sus contextos, las pone en riesgo desarrollando el trauma psicosocial cuyas consecuencias son el duelo traumático, los estigmas socioculturales y el estrés postraumático. Las conclusiones refieren que los contextos de alta vulnerabilidad social, en donde viven las mujeres de diferentes culturas antes de ser viudas, coinciden con las mujeres otomíes para desarrollar el trauma psicosocial. La forma de enfrentar el estrés postraumático radica en la particularidad de las culturas para sobreponerse y enfrentar la viudez; de igual manera, las mujeres indígenas durante siglos han enfrentado discriminación y carencias sociales, en consecuencia, tienen un alto grado de afrontamiento y capacidad de resiliencia.

Palabras clave: duelo; estrés postraumático; estrés psicosocial; resiliencia; viudas

\begin{abstract}
This research analyzes the consequences of the psychosocial trauma experienced by "the white widows)" indigenous women who lost their partners in the explosion of the Petróleos Mexicanos (Pemex) pipelines, on January 18, 2019, in the Municipality from Tlahuelilpan, Hidalgo, Mexico. The study was based, theoretically, on psychosocial trauma and qualitative methodology, with an ethnographic design, applying the techniques of participatory observation, in-depth interviews, and field diary, carried out with a sample of ten women, 30 to 60 years old. The results show that the high social vulnerability suffered by widows in their contexts puts them at risk by developing psychosocial trauma, the consequences of which are traumatic grief, sociocultural stigmas, and post-traumatic stress. The conclusions refer that the women of different cultures live before becoming widows, contexts of high social vulnerability, coincide with Otomi women to develop psychosocial trauma. The way to face post-traumatic stress lies in the particularity of cultures to overcome and face widowhood; Similarly, indigenous women for centuries have faced discrimination and social deprivation; consequently, they have a high degree of coping and resilience.
\end{abstract}

Keywords: duel; post-traumatic stress; psychosocial stress; resilience; widows

López-Pozos, C. (2021). Trauma psicosocial y estrés postraumático en las "viudas blancas" por la explosión de un ducto de gasolina en el Municipio de Tlahuelilpan, Hidalgo. [Psychosocial stress and post-traumatic stress in the "white widows" due to the exploitation of a gasoline pipeline in the municipality of Tlahuelilpan, Hidalgo]. Forhum International Journal of Social Sciences and Humanities, 3(4), 134-148. https://doi.org/10.35766/j.forhum2021.04.03.11

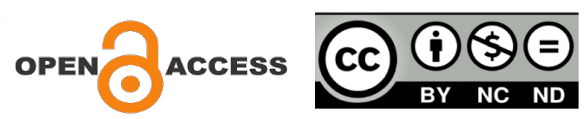




\section{Introducción}

Durante los gobiernos federales del Partido Acción Nacional (PAN) y el último período presidencial del Partido Revolucionario Institucional (PRI) se declaró que el petróleo, conocido como oro negro (Cárdenas et al. 2019), constituía una de las riquezas que sacaría al país de la pobreza. No obstante, los aportes que la empresa paraestatal de PEMEX hacía la economía mexicana, fueron opacados por el despojo realizado por empresas fraudulentas y cárteles que se constituyeron en emporios de riquezas individuales y grupales, mediante la sustracción del petróleo de forma clandestina (Cárdenas et al. 2019; Estrada \& Zavala, 2019).

El robo de combustible es una problemática que se ha extendido y mantenido en diferentes regiones del país desde el 2001 hasta las últimas décadas. En concreto, en el estado de Hidalgo, las tomas clandestinas de petróleo pasaron de 183, en 2016, a 1,064 en 2017; y para 2018, éstas llegaron a ser 2,121; hasta llegar a 2,796 tomas en 2019 (Molina, 2019). La práctica ilegal del huachicol, entendida como el robo de combustible de manera clandestina, es una actividad que se estableció mediante estructuras complejas de bandas criminales que revenden el producto a grandes cantidades y a granel, involucrando a los pobladores de Tlahuelilpan, quienes se beneficiaban del robo como una solución a sus problemáticas económicas sin medir las consecuencias (Estrada \& Zavala, 2019).

Para los gobernantes en turno, el oro negro fue utilizado en el discurso de la esperanza para el desarrollo y sobrellevar la economía mexicana; pero, esta mina, sexenio tras sexenio, se conformó en un conjunto de actividades ilícitas, legales y paralegales que a final de cuentas se derrumbó y quedó como un negocio opaco con infinidad de tentáculos empresariales en declive (Cárdenas et al. 2019).

A finales de 2018, inició el periodo de gobierno de Andrés Manuel López Obrador; el primer mandatario publicó que una de sus prioridades durante su mandato sería el ataque frontal contra el robo de combustible. Una estrategia pertinente en su coherencia discursiva en contra de la corrupción, fue un éxito fugaz que duró poco. El día 18 de enero de 2019, explotó el ducto Tuxpan-Tula, ubicado en el predio de San Primitivo, que se localiza en la zona rural entre los Municipios de Tlahuelilpan y Tlaxcoapan, que se mantenía abierto debido a la extracción del hidrocarburo de manera clandestina. El siniestro causó un sinnúmero de muertos, ocasionando deterioro al medio ambiente, secuelas de dolor, duelo y una acentuada escisión de la población circunvecina de dicha municipalidad.

\section{Marco Teórico}

La experiencia traumática que vivieron las viudas durante y después de la explosión del ducto de gasolina, vulneró sus vidas; por lo que la estabilidad emocional se irrumpió en la confianza de los demás y en las creencias básicas. Dicha catástrofe influyó en las relaciones sociales; como refiere Beristain: "[...] las catástrofes provocan innegables consecuencias e implican un suceso negativo, a menudo imprevisto y brutal que apremia destrucciones materiales y pérdidas humanas, ocasionando un gran número de víctimas y una desorganización social importante" (2000, p. 6).

Además de la desorganización interna de las viudas, el análisis del trauma y de sus características deben ser aplicados al entorno social en que se desenvuelven, con sus propias particularidades de acuerdo a la situación de emergencia que atraviesan; por tanto, la presente investigación retomó como referencia teórica el trauma psicosocial. En este sentido, Martín Baró, plantea el trauma con una perspectiva social: "se trata de un marco conceptual más adecuado para abordar problemas psicológicos y sociales que se derivan de determinados contextos socio-históricos" 
(1990, p. 37). A nivel interno las personas experimentan el trauma como una herida, una vivencia o experiencia, un residuo negativo que las afecta y las deja marcadas permanentemente (Martín Baró, 1990).

Dichas huellas o heridas de la traumatización extrema, se reflejan en un trauma psicosocial, el cual vincula la relación entre las condiciones en las que se ha gestado el trauma — pre-traumáticasy la naturaleza o intensidad del daño causado en consecuencias -pos-traumáticas- es así como el trauma tiene una incidencia de carácter social por su origen y por los efectos que va desplegando en su entorno (Blanco \& Díaz, 2004).

Después de la explosión del ducto de gasolina en el municipio de Tlahuelilpan, Hidalgo, diferentes medios de comunicación dieron a conocer los decesos de los pobladores que sufrieron el suceso, dejando a un lado el padecimiento psicosocial del trauma que vivieron las viudas y las consecuencias para su salud mental, desistiendo esta situación en el anonimato. Al respecto, Jeitani (2019) afirma que después de que se brindó el apoyo moral y social en un inicio a la población de Tlahuelilpan, se dejó de lado el dolor y duelo de las mujeres que perdieron a sus esposos, quienes siguen sin poder reponerse del duro golpe de haber perdido a sus esposos.

Es necesario replantearse las siguientes interrogantes: ¿Qué daños psicosociales presentan las viudas a nivel externo? ¿Cómo viven las viudas el sufrimiento psíquico del TPT a nivel interno? ¿Cuáles son las estrategias de resiliencia y afrontamiento positivo que desarrollaron para enfrentar la vida como viudas? Cuestionamientos que se irán respondiendo a lo largo de la descripción de esta investigación.

El propósito de este estudio, es desentrañar las consecuencias del trauma psicosocial que viven las mujeres indígenas de la cultura otomí que perdieron a sus parejas en la explosión del ducto de gasolina, cuya relevancia radica en plantear cómo la delincuencia del huachicol es un factor de riesgo para toda la población debido a condiciones de alta vulnerabilidad que padecen los pobladores de Tlahuelilpan, quienes se exponen a padecer una catástrofe que altera tanto la salud física como la psíquica, problema que hasta la fecha no se ha resuelto.

Este estudio tiene como objetivo general conocer las consecuencias del estrés psicosocial que viven las mujeres indígenas que perdieron a sus parejas en la explosión del ducto de gasolina . Los objetivos específicos que se plantean son: 1) describir las características contextuales del trauma psicosocial, pre-traumáticas y pos-traumáticas que padecen las mujeres viudas después del evento; 2) puntualizar la práctica de los rituales religiosos como un componente de resiliencia y afrontamiento para sobreponerse a la pérdida.

\section{Contexto social}

El Municipio de Tlahuelilpan, Hidalgo, cuenta con una extensión territorial de $28.18 \mathrm{~km}^{2}$, asentado en la altiplanicie mexicana y la región geo cultural del Valle del Mezquital, ubicado en la región del río Pánuco, a la cuenca del río Moctezuma, donde cruza a través de éste el río Tula (Molina, 2019). Su población asciende a 19389,9439 hombres y 9950 mujeres; a nivel general la mayoría de los hogares están dirigidos por varones y en menor escala por una mujer.

El 43\% de la población, tanto del medio rural como urbano, se identifican como indígenas, del grupo étnico Otomí, aunque no hablen ninguna de las lenguas originales otomí o náhuatl. Los habitantes de la comunidad confirman sus costumbres y valores, tales como: solidaridad, vínculos familiares de apego, permanencia marital y la pertenencia a la religión católica, aspecto que favorece la práctica de diferentes rituales religiosos, fiestas patronales, festividades del día de los muertos, 
entre otras tantas celebraciones de índole comunitario que benefician su identidad, a pesar de la invasión moderna del interior del país y de la influencia extranjera por el amplio sector varonil que emigra a los Estados Unidos (INEGI, 2015a).

La ubicación territorial de la localidad marca su colindancia con los municipios de Tezontepec, Mixquihuala de Juárez, Tepetango, Tetepango, Tlaxcoapan y Tezontepec de Aldama; la extensión del territorio de los diferentes municipios permite rodear la refinería de Tula. Además, tanto, Tlahuelilpan como Tlaxcoapan, están ubicados a unos 13 kilómetros de la refinería por donde atraviesa el ducto de gasolina de Tuxpan a Tula. Por tal motivo, la perforación de diferentes ductos en cualquier parte del territorio es frecuente, sin que se tenga ninguna precaución del cuidado humano y ambiental (Molina, 2019).

La población económicamente activa de 12 años y más, entre el periodo 2000 y 2015, la oferta de trabajo tuvo un incremento de 46.4 a $47.2 \%$. Del total de este sector productivo el 19\%, se ocupa en el sector primario, el $22.9 \%$ en el secundario y el $57.5 \%$ en el terciario; de acuerdo con estos datos la medición de la pobreza a nivel municipal es de $55.5 \%$ y 7\% vive en pobreza extrema, lo que representa una alta vulnerabilidad social en todos sus habitantes (INEGI, 2015b).

A nivel general, el estado de Hidalgo presenta un rezago educativo en población mayor de 16 años el 32.5\%, lo cual implica el bajo aprovechamiento de la población adolescente, la deserción y el ausentismo (CONEVAL, 2010; Plan de desarrollo Municipal, Tlahuelilpan, 2016-2020). En este contexto, las redes locales de los huachicoleros encontraron el ambiente apropiado para hacer una estructura clandestina de protección y contubernio del robo de combustible (Cárdenas et al. 2019), involucrando a jóvenes y adolescentes, sin tener ningún control de seguridad. Otro aspecto que incide en la alta vulnerabilidad de esta población es la falta de los servicios médicos, solo cuenta con dos centros de atención externa de la Secretaría de Salubridad, caracterizada por la falta de infraestructura médica respecto a la prestación de servicios hospitalarios, tanto de forma general como especializada.

Los estudios basados en evidencia de tragedias humanas ocasionadas por los desastres naturales - así como los causantes por los errores humanos - siempre dejan signos de muerte y destrucción (Medina, 2015). Una buena parte de la literatura existente sobre este tipo de situaciones se centra en el Trastorno de Estrés Pos-Traumático TEPT (Medina, 2015; Manual Diagnóstico y Estadístico de Trastornos Mentales, DSM-IV-TR, 2002; Henry \& Sekar, 2013). Asimismo, el trauma social, independientemente de las causas, genera vulnerabilidad y desestabilización socioemocional.

Las viudas son más sensibles en los primeros meses de duelo, pero una vez que se recuperan se sobreponen a las adversidades como veremos en los siguientes estudios. Mukimulhasan et al. (2013), muestran cómo las consecuencias psicosociales entre mujeres viudas indias y musulmanas fueron diagnosticadas con el trastorno de duelo prolongado, además de padecer depresión mayor y trastorno de estrés postraumático (TEPT). Sin embargo, las viudas que reportaron mayor impacto emocional del trauma fueron las mujeres hindúes, en comparación con las viudas musulmanas.

El 15 de marzo de 2013 en la comunidad de Nativitas (Tlaxcala, México), la población celebraba una fiesta religiosa con quema de cohetes; esta festividad causó una explosión afectando a las personas que participaban de manera colectiva. El trauma dejó secuelas tanto físicas como psíquicas (miedo, culpa, presión y tristeza); estos síntomas se encuentran asociados al contexto psicosocial que suscitó el incidente, interpretándolo con un sentido religioso, de súplica, reparación y resiliencia para toda la comunidad (López-Pozos, 2014).

Otras circunstancias socioculturales presentan las jóvenes viudas de Banso, en Camerún; en ellas se presentan elementos psicológicos del trauma y el dolor están asociados a los estilos de duelo y rituales en donde sacrifican inadvertidamente sus responsabilidades parentales. Éstas son 
presionadas por la familia del esposo para matrimoniarse con el hermano del difunto, sometiéndose al control de la familia; y ante lo cual muestran síntomas psíquicos asociados a los patrones de dolor intuitivo y combinado como parte del TEPT que padecen (Kinyuy, 2016).

El dolor y el sufrimiento se hace presente, también, en las viudas de Sundarban Delta, India, cuyos maridos murieron por ataque de tigres de la región; las secuelas del incidente, así como las consecuencias del duelo, el enfrentamiento del estigma cultural y la discriminación, tienen impacto en la salud mental de estas mujeres. De las 65 viudas, el 44\% padecía una enfermedad mental, asociada con las creencias y prácticas socioculturales locales, junto con una multitud de cicatrices psicológicas posteriores al trauma, privación, abuso y explotación (Chowdhury, 2016).

Atwal (2017) explora los traumas culturales y ciertas cuestiones que determinaron las pensiones de las viudas de la India que perdieron a sus maridos en las Guerras Mundiales a lo largo del siglo XX. Estas viudas, tanto indias como británicas, lideraron las consecuencias del Estado colonial no son tratadas con paridad a las viudas de los soldados oficiales que murieron luchando en las guerras en 1962, 1965, 1971 y 1999 con China y Pakistán, respectivamente; mientras las primeras son consideradas viudas del imperio y éstas últimas son viudas de la nación, tanto unas como otras, enfrentan un trauma cultural estructurado por ideologías religiosas, costumbres culturales, leyes ancestrales y castas.

En cuanto a la depresión como síntoma del TEPT, prevalece entre las mujeres viudas mayores de más de 55 años de edad, en comparación con las mujeres jóvenes del medio rural de Kerala, India. Además de la influencia del contexto social que viven las mujeres en las aldeas, dónde se acentúan todavía más aspectos culturales acerca del deber de la mujer en esos ámbitos (Archana et al. 2017).

En las viudas tamiles de la Provincia Oriental de Sri Lanka, se evaluaron las relaciones ligadas a los problemas con la guerra y los indicadores de angustia psicológica, consecuencia de los desastres, tales como las cogniciones postraumáticas y síntomas depresivos culturalmente específicos. Resultando que, las relaciones basadas en lazos familiares pueden favorecer mejores niveles en la salud mental de las viudas (Lambert et al. 2017).

Kim et al. (2017) y Pragya et al. (2018), analizaron el trastorno cultural del duelo complejo persistente y del duelo temprano, en las viudas nepalíes las cuales narran cómo entienden el dolor y cómo construyen el duelo complicado persistente. El estudio referido, indica que los factores estresantes tanto económicos y familiares son determinantes para que se prolongue y complique el duelo, presentando reacciones psicológicas negativas en respuesta al duelo y la ideación suicida.

Sin embargo, el concepto de duelo complejo persistente se identificó con los efectos psicológicos coexistentes de la violencia armada, tortura, discriminación social y dificultades económicas (Worden, 1997), con una duración aproximada de un año, más que los duelos normales; además de las respuestas somáticas que perturbaron a las viudas. Tanto las viudas de la guerra de la India, así como las viudas de guerra, del suroeste de Nigeria, revelan que su condición es una experiencia traumática, porque no solo pierden al marido, sino ello implica el detrimento significativo de estatus social e importancia en sus familias; además, son tendientes a aceptar responsabilidades por la crianza de sus hijos, el cuidado de los ancianos del clan, así como soportar el hostigamiento sexual y las agresiones en un contexto de dominio (Omozusi et al. 2018; Sharma \& Lehal, 2018).

En otras regiones, las viudas de la guerra de las Fuerzas de Defensa de los Beduinos de Israel, revelan las exclusiones y el sometimiento por factores estructurales familiares de orden patriarcal. La poligamia refleja la continua opresión y exclusión de estas mujeres que una vez que se quedan solas deben permanecer con la familia de origen del marido, obligadas a mantener la acción del marido 
caído como símbolo nacional, perpetuando los valores culturales de patriotismo y heroísmo, según (Bokek-Cohen \& Ben-Asher, 2018).

En Chihuahua (México) se encuentra Ciudad Juárez, la cual es una de las ciudades más controvertidas por la violencia; este territorio se ha convertido en un espacio social de guerras entre grupos de narcotraficantes y policías. Como consecuencia, han quedado en el abandono un numeroso grupo de mujeres viudas, que presentan el síndrome del TEPT, con síntomas de hipervigilancia, ideación recurrente asociada al asesinato de su pareja, así como indicios del duelo traumático persistente debido a la constante inseguridad y peligro que viven junto con sus hijos (Aguirre, 2018).

En otros contextos, pero en condiciones similares, se encuentran las viudas del genocidio en el distrito de Nyarugenge, Ruanda, que recibieron apoyo para superar el trauma y evaluar el incremento del TEPT. Los hallazgos indicaron que las intervenciones y el soporte recibido disminuyeron notablemente la sintomatología del TEPT, a nivel moderado, pero persisten las consecuencias del trauma debido a las condiciones de pobreza extrema (Hategekimana et al. 2019).

Finalmente, las viudas que han perdido a sus parejas por diferentes circunstancias en Jordania, recibieron apoyo social de parte del Estado. Los resultados revelaron que el nivel de apoyo social percibido entre las mujeres viudas fue moderado, en comparación con el sostén más alto recibido de la familia de origen, para sobreponerse a las adversidades que enfrentan como mujeres solas (MansourGhaith et al. 2020).

Con base en lo expuesto anteriormente podemos aseverar que en su totalidad las mujeres de India, África, Asía y América; antes de ser viudas vivían condiciones socioculturales de alta vulnerabilidad como guerras, genocidios, pobreza, violencia, tortura, discriminación racial y que la manifestación del Trastorno de Estrés Postraumático, es una expresión de malestar social y personal con una diversidad de sintomatologías, connotaciones y significados particulares de cada cultura que aumenta el sufrimiento y lacera la salud mental de estas mujeres.

\section{Metodología}

Tipo de estudio

Esta es una investigación exploratoria, de tipo cualitativo, con un diseño Etnográfico, mediante la cual buscamos las consecuencias del trauma psicosocial, el significado y la interpretación que las viudas le dieron al evento traumático de la explosión, ante la pérdida de sus parejas.

La integración a la comunidad de Tlahuelilpan se llevó a cabo con el apoyo de los líderes religiosos y de dos informantes claves originarios del lugar de estudio con un conocimiento real de las condiciones sociales del lugar y que fueran líderes en algún cargo de índole comunitario (un hombre y una mujer); durante un mes se llevó a cabo la inmersión en el campo, participando en la observación participante e iniciamos las primeras entrevistas piloto.

Posteriormente, seleccionamos intencionalmente a las viudas que habían perdido a su pareja en el incidente, con la finalidad de que el grupo fuera de la misma comunidad, lo más homogéneo posible, éste quedó constituido por 10 mujeres de 30 a 60 años de edad, con una escolaridad de primaria, secundaria y universidad, de ocupación comerciantes, campesinas, empleadas y profesionistas (Hernández-Sampieri et al. 2014).

Se aplicaron tres entrevistas en profundidad en diferentes tiempos y circunstancias. Y una vez aplicadas se determinaron los siguientes ejes centrales: 1) contexto que favoreció el robo de combustible; 2) el impacto de la pérdida del esposo en esas circunstancias y su significado; 3) 
sufrimientos que padecieron durante la perdida y cómo lo expresan; 4) problemas que enfrentan desde la pérdida del esposo y 5) rituales religiosos que han practicado como estrategia resiliente para superar el duelo.

Al finalizar las entrevistas, se transcribieron y complementaron con las anotaciones de la observación del diario de campo. Posteriormente, se realizó una codificación inicial, leyendo cuidadosamente los datos, a fin de etiquetar las ideas y los temas analíticamente significativos. Inmediatamente, se identificaron las categorías que describían hallazgos que mostraban los objetivos de investigación que fueron planteados: describir las características contextuales del trauma psicosocial, pre-traumáticas y pos-traumáticas que padecen las mujeres viudas después del evento.

Las codificaciones se hicieron de línea en línea a través de los datos y etiquetas creadas inicialmente. Después, se analizaron las etiquetas de los datos y se codificaron por segunda vez. Finalmente, se definieron y discutieron las categorías matrices a fin de esclarecer los hallazgos que anotamos como resultados finales (Martínez, 2007).

\section{Resultados}

Los resultados responden a los planteamientos iniciales de conocer las consecuencias del trauma psicosocial que vincula, por un lado, las condiciones en que se ha generado el trauma (pretraumáticas), y por el otro, la intensidad del daño en consecuencias (pos-traumáticas), que viven las viudas de los “quemados” después de la explosión (Blanco \& Díaz, 2004).

\section{Condiciones de alta vulnerabilidad}

Las condiciones previas al evento de la explosión en las que vivían las mujeres de la comunidad de Tlahuelilpan, nos muestran una alta predisposición a la vulnerabilidad psicosocial caracterizada por las condiciones sociales y origen étnico, así como a la falta de herramientas de adaptación que les impide incorporarse al desarrollo y acceder a mejores condiciones de bienestar. Lo cual influyó para mayor predisposición a padecer problemas relacionados con la salud psicosocial que implica la exposición a mayor riesgo, y en que el comportamiento se vea expuesto a acontecimientos más alarmantes que puedan provocar consecuencias negativas para la salud, aspecto del que dan cuenta la indagación de nuestros informantes:

Aquí todos los días era una fiesta, subían y bajaban camionetas, motos, venta de comida, por todas partes "había economía"; los morros eran los que más consumían, y también creció la drogadicción y la prostitución, pero vea, ahora nada, y qué hicimos, no se hizo nada para el pueblo, fue un dinero que se fue en puro vicio. (Geno, comunicación personal, 17 de julio de 2019).

Las comunidades pertenecientes al Municipio de Tlahuelilpan con alta vulnerabilidad, desempleo, subempleo, con rezago económico, pobreza y marginación son el espacio social adecuado para la captación de los jóvenes y adolescentes que participan en las actividades del huachicol. La fuerza de trabajo del lugar laboraba como choferes, repartidores, halcones y cobradores, siendo población vulnerable que participaban en las actividades clandestinas, cuya organización paralegal está constituida por comerciantes que constituyen y estructuran el mantenimiento de dicha actividad (Molina, 2019). 
Las comunidades que por generaciones han vivido carencias y marginación social, dan cuenta que, la ilegalidad, aunque sea riesgosa y peligrosa, la asumen como una forma de vida, a fin de reparar las carencias permanentes que se viven a nivel familiar como lo testifica el siguiente testimonio.

Mi hijo mayor dejo de trabajar y empezó a tener más dinero, luego me dijo mi marido que iba a trabajar con mi hijo de chofer de pipas que repartían la gasolina, después me convenció para que yo repartiera la gasolina en las casas y acepté. Uno va teniendo su dinerito, qué sabíamos que no iba a durar mucho. El segundo hijo, como vió que se ganaba bien, dejó la prepa y también es chofer. Pero ahorita con lo que pasó, dice mi marido que esperemos a que se tranquilice todo, solo que ahora tengo anemia y ya no puedo hacer muchos encargos. (Lucía, comunicación personal, 25 de julio de 2019).

Para las personas que pertenecen a las redes clandestinas el riesgo y la alteración de la salud no son motivo suficiente para dejar las actividades ilegales, la mayor compensación se reditúa por el resarcimiento económico y consideran que es una actividad necesaria para paliar sus necesidades básicas.

Después de la explosión, esta región quedó contaminada por los hidrocarburos procesados del petróleo, debido a excavaciones clandestinas que han afectado el suelo y el agua. Además del paso de las aguas negras y los efectos colaterales de la contaminación ambiental, dicho contexto se torna en una serie de circunstancias que afectan a la población en su salud, tanto física, como mental (Molina, 2019). Estos datos confirman que los ambientes en que se generó el trauma se constituyó en un contexto sociohistórico que predispuso a las viudas blancas a padecer mayores consecuencias con el incidente de la explosión (Martín Baró, 1990).

Y ante la incapacidad de resolver las condiciones aumenta la vulnerabilidad, causando un estado emocional caótico, con mayor predisposición a padecer desastres y accidentes graves, situaciones que pueden afectar el desarrollo de la identidad personal (Medina, 2015). Que debilitada y con un afrontamiento débil desarrollaron un duelo traumático, más prolongado.

\section{Duelo traumático}

La explosión del ducto de gasolina en la comunidad fue causada por la acción humana, que perdió el límite de lo consciente y entró en un nivel inconsciente de masa; originando una catástrofe que causó un duelo traumático, en las viudas, entendido como "un fenómeno vivo, doloroso que ocasionó la muerte de varias personas en una situación sorpresiva, inesperada e injusta" (Worden, 1997, p. 23). Además, se produjo una desestructuración del yo que redunda en debilidad por la herida psicológica de perder a la pareja y permanecer como viuda de manera intempestiva.

Situación de crisis que está asociada a una reacción severa de duelo traumático, y al padecer exceso de violencia emocional se quiebra el funcionamiento mental (Dorsch, 1991) que, ante la inesperada perdida de la persona amada, produce un trauma psíquico, retroalimentado por una estimulación intensa que rompe con la barrera protectora de los mecanismos de defensa, produciendo que el psiquismo sea desbordado por la debilidad del Yo, lo cual propicia el desarrollo del TEPT.

El duelo es el proceso psicológico normal que se produce a partir de la pérdida por la muerte de una persona querida y tiene una duración limitada en el tiempo, aproximadamente entre uno o dos años (Lundin, 1984). Al respecto, cuando se entrevistó a las viudas, de este estudio, solo habían pasado cinco meses de la tragedia y ellas presentaban mayores niveles de embotamiento y fuertes sentimientos de vacío, lo cual nos indica la experiencia de un duelo inmediato caracterizado por reacciones perturbadoras confusas, así como la incredulidad de creer en el incidente, asociado a una constante sensación de presencia de la persona fallecida. 
En tales casos, las viudas expresaron sentirse ansiosas y deprimidas, ya que el duelo traumático inesperado fue más doloroso, manifestado con crisis severas de llanto, tristeza, ira, coraje, dolor, culpa, remordimiento, ansiedad, ambivalencia, soledad y fuertes sentimientos de desamparo. Dichos signos y síntomas en su conjunto nos muestran la crisis del duelo traumático inesperado (Worden, 1997; Lundin, 1984), tomando en cuenta el transcurso del tiempo del suceso y el momento en que se realizaron las entrevistas.

Tabla 1. Descripción de los síntomas del duelo traumático.

\begin{tabular}{|c|c|}
\hline Emoción & Testimonios \\
\hline $\begin{array}{l}\text { Estado de confusión/ } \\
\text { ambivalencia }\end{array}$ & $\begin{array}{l}\text { "Me sentía muy mal, no lo aceptaba, no lo comprendía, fueron días y } \\
\text { noches de mucha angustia." (Paz) } \\
\text { "Empecé a gritar, cómo era posible [se lo llevó su padre], no entendía que } \\
\text { me decían, lo único que deseaba era volver a verlo." (Jovita) } \\
\text { "Yo misma no podía ni pensar, me preocupaban mis niños qué iba a ser, si } \\
\text { todo me lo daba mi esposo." (Pilar) }\end{array}$ \\
\hline Ira/Coraje & $\begin{array}{l}\text { "Sentí mucho coraje, porque no tenía por qué estar ahí." (Tere) } \\
\text { "Yo misma no supe cómo podía haber sucedido, sentía rabia." (Lupita) } \\
\text { "Pues en los momentos de desesperación sentí rabia por qué tenía que } \\
\text { pasarle a él." (Leonor) }\end{array}$ \\
\hline Dolor & $\begin{array}{l}\text { "Es un dolor inexplicable, de la noche a la mañana tu vida cambia." (Rosita) } \\
\text { "Yo siento que mi vida se paralizó, es como un hueco en el alma." (Geno) } \\
\text { "Cuando te falta el marido es como un vacío, y nunca lo vas a llenar." } \\
\text { (Jovita) } \\
\text { "Es un dolor de muerte, nada te consuela y llorando me consuelo." (María) }\end{array}$ \\
\hline
\end{tabular}

Llanto/Cansancio

"Lloraba día y noche, nada me consuela, estoy sola y en las noches lloro." (Paz)

"He llorado desde que no está mi marido, me alegraba la vida. He sufrido tanto, que me siento cansada, no tengo ganas de hacer nada." (Pilar)

"Lloraba, lloraba más mucho, sólo pensaba en mis hijos y cómo los iba a sacar adelante." (Leonor) 


\section{Tristeza}

Viuda
"Te sientes triste, tu casa vacía, su lugar, sus cosas y las ves, te sientes más triste." (Pilar)

"Con la tristeza no comía, no dormía y todavía todo lo que tuvimos que pasar." (Paz)

"Cuando te pones a pensar que la vida te lo quitó, quisieras morir por tanta tristeza, pero están tus hijos y te necesitan, me consuelan lloran conmigo porque nos hace falta." (María)

"Ser viuda es un título que aún me cuesta aceptar. Pues considero estar sola, desprotegida." (Pilar)

"Te quedas desprotegida, y te cambia la vida [...]. Los hombres te ven más necesitada." (Lupita)

"Quedarme sin él fue muy extraño, yo no trabajaba porque él nos proveía siempre la comida, y siempre me acompañaba. "(Leonor)

"Yo me siento sola, el marido me falta, aunque tenga el apoyo de mis suegros no es lo mismo." (Jovita)

Fuente: Elaborada por la autora para la presente investigación.

Dentro de los valores de la cultura otomí, la vida en familia es muy importante; la pérdida de la pareja es irreparable y el dolor psíquico de la separación definitiva se muestra en estados de tristeza con todos los síntomas de la depresión y, sobre todo, las implicaciones de ser viuda son percibidas con acoso y discriminación, aunque estas condiciones permitieron afrontar su nuevo rol ante la sociedad.

El proceso de duelo traumático, implica un tránsito doloroso vivenciado en torno a las creencias de la propia cultura que refieren la gran diversidad de signos y rituales en torno a las concepciones y opiniones sobre la muerte, así como la esperanza de reencontrarse con el difunto cuando la viuda muera. Las expresiones culturales del duelo en el contexto rural identificado con los valores de la cultura Otomí, son un referente para entender como cada emoción es muy significativa ante la pérdida del marido. En este ámbito, la vida de pareja se concibe para toda la vida y la construcción de la identidad se va dando en razón de los años de convivencia como esposos.

Las mujeres viudas han tenido que confrontarse con otra realidad, la nueva organización familiar implica crisis de angustia, asociadas con el miedo e incertidumbre en el futuro, situación que las ha hecho capaces de desarrollar mecanismos de afrontamiento y sobreponerse en el medio social donde se desarrollan. Es necesario comprender la conexión entre la cultura y el duelo desde la propia cultura de la doliente y detectar cuáles son las diferencias culturales, respecto a la generalidad de la muerte porque no se puede catalogar que todas las muertes tienen el mismo significado.

Para estas mujeres, la muerte es una oportunidad de reencontrarse con su pareja cuando ellas mueran. Las sorprendentes formas en que el duelo se vive en la cultura Otomí en comparación con otras culturas, tiene sentido en sus propios términos, incluso si son absurdos desde la perspectiva de las culturas occidentales (Rosenblatt, 2008). 


\section{Estigmas socioculturales y estrés postraumático}

Aunado a las exigencias del nuevo rol, el total de las mujeres entrevistadas muestran que en el medio sociocultural al que pertenecen, han sido señaladas, $\mathrm{y}$ fuera de su contexto tuvieron que asumir los señalamientos que les hicieron en las instituciones de salud, donde sus esposos se encontraban hospitalizados, como refieren los siguientes testimonios:

Llegamos al hospital, el mismo personal nos decía: -eso les pasó por ladrones, si su esposo era tan inocente, ¿entonces por qué se quemó? -.

El modo de tratarnos, como nos miraban, el cuchicheo del personal del hospital [...], fue muy discriminatorio, porque piensan que en verdad somos ignorantes y no entendíamos lo que nos decían. (Paz, comunicación personal, 19 de julio de 2019).

Después de todo, el calvario que tuvimos que pasar, el interrogatorio de por qué estaba mi esposo, y de todas las maneras aparecíamos como cómplices y como rateros, aunque no teníamos más que la triste realidad de estar quemados. (Leonor, comunicación personal, 20 de julio de 2019).

Afirmaciones que se lanzan sin reflexionar acerca de las circunstancias y el contexto que llevó a la pérdida de vidas humanas, señalamientos que afectaron la autoestima de las viudas, de ser cómplices o de fomentar el robo en sus familias. Otra situación de discriminación fue que dentro de las comunidades es muy importante la presencia del marido la vida de pareja tiene mayor incidencia social cuando los maridos viven. Y ante la falta, estas mujeres tuvieron que sobreponerse a los señalamientos y al acoso, para seguir adelante y empezar a reorganizar su vida. Circunstancias que favorecieron el desarrollo del TEPT, como afirma Damacene et al. (2018), las personas expuestas a traumas, incluyendo desastres naturales, accidentes y violencia interpersonal, son más vulnerables a padecer dicho síndrome.

Sin embargo, aunado al trauma del accidente las mujeres atenuaron el TEPT al ser expuestas públicamente, discriminadas y estigmatizadas al enfrentar los procesos legales, declaraciones, reconocimientos, juicios, aniversarios y rituales, así como el acoso persistente de representantes de diferentes medios de comunicación (Medina, 2015). Que ante los señalamientos nuevamente se revictimizaban sin tener los recursos psíquicos, legales y económicos para sobreponerse a las condiciones de estrés que vivían. Como refiere el siguiente testimonio:

No te puedes olvidar, todo te lo recuerda, en todos los trámites que he tenido que hacer para recuperar los restos del cuerpo, y el papeleo de mis hijos. Cada día lo vives como martirio [...], llega el momento que la tristeza te acaba y te quieres morir... (Pilar, comunicación personal, 20 de julio de 2019).

La re-victimización de memorar constantemente las condiciones del fallecido, abren las heridas psíquicas retroalimentadas por las expectativas, y en ocasiones la curiosidad del personal de instituciones gubernamentales que reiteradamente marcaban la tortura de revivir los momentos traumáticos, ante lo cual la reminiscencia del TEPT se estableció permanentemente en depresión manifestada en sentimientos de tristeza, llanto y agotamiento, causando un deterioro psicosocial y alteración en la calidad de vida y la salud mental de las viudas (Grubaugh et al. 2005).

\section{La religiosidad, resiliente de sanación}

Ante la pérdida del marido, y las consecuentes vulnerabilidades sociales, las viudas blancas es una expresión que refiere el sentido en el que fueron señaladas como cómplices, y eso no se pudo 
demostrar ante las autoridades. Con el cúmulo de experiencias traumáticas como se han descrito la capacidad de afrontamiento en las mujeres aumentó, sobreponiéndose a las adversidades vividas y la perdida, siendo más resilientes ante la certeza que marca la vida comunitaria del pueblo Otomí en la relación que tienen con lo divino y trascendental, desde su propia cosmovisión.

La repetición constante de diferentes rituales dentro de la religiosidad popular, sirven como catarsis a tanta ausencia y desprecio que han vivido por siglos. De esta forma las viudas realizan diversas prácticas etnoculturales, en un intercambio permanente con la divinidad, entre súplicas y peticiones que se constituye en la única estrategia resiliente de bienestar para la salud de estas mujeres:

Asistimos a misa, al principio no queríamos volver a pisar la iglesia porque decíamos que Dios nos falló al llevárselo, luego entendimos las cosas, y ahora vamos a misa para que lo cuide y nos dé fuerza en nuestro corazón y siempre le ponemos una veladora. (Paz, comunicación personal, 20 de julio de 2019).

Lo que hago es ir a misas, rosarios, recordatorios. Visitar la capilla. (Rosita, comunicación personal, 20 de julio de 2019).

Participo de oraciones en mi soledad... pero también me voy a retiros... (María, comunicación personal, 20 de julio de 2019).

Las creencias y prácticas religiosas que las dolientes realizan, conjuntamente, han podido reparar y construir su identidad como viudas, proveedoras y jefas de familia, a pesar de enfrentar las secuelas del TEPT, que después de rememorar los diferentes rituales de sus creencias, encuentran alivio. Puesto que, ante las condiciones carentes revitalizan sus rituales como psicoterapia curativa, favoreciendo el afrontamiento y disminución del malestar, mismo que les sirve como un paliativo para calmar sus heridas y retomar la cotidianidad, así como las responsabilidades en la nueva estructuración familiar.

\section{Discusión y conclusiones}

La marginación y las carencias sociales que han vivido las viudas desde sus contextos, las exponen a vivenciar todo tipo de peligros y catástrofes, tal y como se vivió en la explosión del ducto de gasolina. Además, la permanente contaminación ambiental por el robo de combustible, entre otros tantos eventos traumáticos, mismos que se han constituido en el ambiente para el desarrollo del trauma psicosocial, así como la desorganización social que han tenidos que experimentar (Beristain, 2000; Blanco \& García 2004; Martín-Baró, 1990).

Las evidencias confirman que después de la explosión, las viudas blancas presentan características psicológicas de duelo traumático inesperado, expresadas en emociones de tristeza, ira, coraje, dolor, llanto, estado de confusión/ ambivalencia, miedo y culpa; síntomas que se acentuaron, ante los estigmas socioculturales y la discriminación, dando origen al estrés postraumático como consecuencia del trauma psicosocial.

Un aspecto que enriquece este estudio es corroborar que, aunque las viudas provengan de diferentes culturas, países, espacio social, circunstancias históricas, estas mujeres antes de ser viudas viven en condiciones de alta vulnerabilidad social, lo cual coadyuva a exponerse a mayores riesgos, circunstancias que constituyen el desarrollo pretraumático del trauma psicosocial. Aunque el desarrollo del síndrome postraumático tenga diversas manifestaciones asociadas a sus entornos culturales. (Chowdhury, 2016; Kinyuy Lange, 2016; Atwal, 2017; Archana et al. 2017; Kim et al. 
2017; Lambert et al. 2017; Omozusi Omosefe et al. 2018; Pragya et al. 2018; Sharma \& Lehal, 2018; Bokek-Cohen \& Ben-Asher (2018).

La presente investigación, muestra que la eficacia de la resiliencia de las viudas blancas del grupo étnico Otomí, radica en la práctica de los rituales de la religiosidad popular como herramienta de resiliencia positiva, hallazgo similar en la investigación realizada por López-Pozos (2014) con población tlaxcalteca. Lo cual demuestra que, los pueblos originarios han sido y son desde hace siglos los actores claves en la resiliencia, soportando todo tipo de malestares. A pesar de tanto sufrimiento psíquico y económico, se rehacen por sus valores culturales e identidad, independientemente de la forma en cómo los estigmatice la población mestiza.

Sin embargo, consideramos que este estudio deja un vacío en relación a identificar si en los problemas posteriores que enfrentan las viudas persiste el TEPT, a pesar de las prácticas resilientes curativas. De igual forma, analizamos que los hallazgos sobre el duelo traumático inesperado no se pueden generalizar a todas las mujeres viudas indígenas, porque la muestra es muy pequeña y, por ende, las interpretaciones culturales de cada grupo étnico son muy diversas. Empero, deja indicios para que se realicen proyectos de intervención psicosocial en la prevención de la salud mental, la mejora del ambiente y la oportunidad de propiciar el desarrollo sostenible en las comunidades indígenas (Luna-Nemecio et al. 2020).

Finalmente, las diferencias culturales no solo promueven diferentes construcciones del Yo, sino contrastes en cuanto a concepciones culturales propias de cada grupo étnico, por lo que es un error que, desde posturas particulares, se generalicen conceptos universales. Sin embargo, el hecho de habitar un mundo global nos orienta a entender desde diferentes contextos el sufrimiento y laceración en la salud mental que padecen las mujeres viudas, víctimas de distintos incidentes violentos, de guerra y de la naturaleza que conduce al desarrollo del trauma psicosocial y Estrés Postraumático, TEPT.

\section{Referencias}

Aguirre, L. M. (2019). Las narrativas de las viudas en Ciudad Juárez: una estrategia metodológica para la intervención del trabajador social en contextos de violencia criminal. Revista de Trabajo Social, 2(18), 117-138. http://www.revistas.unam.mx/index.php/ents/article/view/69820

Archana, P. S., Das, S., Philip, S., Rekha, R., Philip, R. R., Jhoseph, J., ..Punnoose, V. P., end Lalithambika, D. P. (2017). Prevalence of depression among middle aged women in the rural area of Kerala. Asian Journal of Psychiatry, (29), 154-159. https://doi.org/10.1016/j.ajp.2017.05.016

Atwal, J. (2017). Cultural Trauma and Welfare for War Widows in India. Croatian Political. Science Review, 4(1-2), 52-73. https://1bestlinks.net/uxchz

Beristain, C. M. (2000). Apoyo psicosocial en catástrofes colectivas: de la prevención a la reconstrucción. Fondo Editorial Humanidades. https://1bestlinks.net/cu2cj

Bokek-Cohen, Y. \& Ben-Asher, S. (2018). The Double Exclusion of Bedouin War Widows. International Journal on Minority and Group Rights, (25), 112-13. https://doi.org/10.1163/15718115-02501007

Blanco, A. \& Díaz, D. (2004). Bienestar social y trauma psicosocial: una visión alternativa al trastorno de estrés postraumático. Clínica y Salud, 15(3), 227-252. http://www.redalyc.org/articulo.oa?id=180617834001 
Cárdenas, C., Grunstein, M. J., Saxe-Fernández, A. L., Pérez, S. K., y Rico, I. (2019). Oro Negro.

En la encrucijada. México: Universidad Autónoma de México.

Chowdhury, A. N., Mondal. R., Brahma, A., end Biswas, M. K. (2016). Ecopsychosocial Aspects of Human-Tiger Conflict: An Ethnographic Study of Tiger Widows of Sundarban Delta, India.

Environmental Health Insights, (10), 1-29. https://doi.org/ 10.4137/EHi.s24899

CONEVAL (2010). Plan de desarrollo Municipal, Tlahuelilpan, 2016-2020. https://n9.cl/uxvl

Estrada, A. y Zavala, M. (2019, 22 de enero). Hidalgo se convirtió en paraíso del huachicol. El

Universal. https://1bestlinks.net/0nZMa

Damascene, J., Uwera, G., Hategeka, M., Kayitesi, M. L., Kaputu Kalala Malu, C. \& Hategeka, C. (2018). Burden of post-traumatic stress disorder acute exacerbations during the commemorations of the genocide against Tutsis in Rwanda: a cross-sectional study. The Pan African Medical Journal, (30), 2-8. https://doi.org/10.11604/pamj.2018.30.216.15663

Dorsch, F. (1991). Diccionario de psicología. España: Herder.

Grubaugh, A. L., Magruder, K., Waldrop, A., Elhai, J., Knapp, R., Subthreshold, F. (2005).

Subthreshold PTSD in Primary Care: prevalence, psychiatric disorders, healthcare use, and functional status. Journal of Nervous and Mental Diseases, (193), 658-664. https://doi.org/10.1097/01.nmd.0000180740.02644.ab

Hategekimana, P., Macharia, S. M., \& Urbanus, M. N. (2019). Avega Interventions and Post Traumatic Growth Among Genocide Widows in Nyarugenge District, Rwanda. The International Journal of Social and Development Concerns, 8(3/10), October 32-43. http://ijsdc.org/storage/bcb193e3d1307a4b2360bbccf160a126.pdf

Henry, G.C. \& Sekar, K. (2013). Women Widowed in the Disaster: A Psychosocial Perspective. Artha J Soc Sci, 12(2), 21-32. https://doi.org/10.12724/ajss.25.3 21

Hernández-Sampieri, R., Fernández, C., y Baptista, M. P. (2014). Metodología de la Investigación. Mc Graw Hill.

INEGI (2015a). Catálogo de claves de entidades federativas, municipios y localidades.http://geoweb.inegi.org.mx/mgn2k/catalogo.jsp

INEGI (2015b). Censo General de Población y Vivienda 2000; Censo de Población y Vivienda 2010. Encuesta Intercensal 2015. https://n9.cl/oyja

Jeitani, S. M. (2019). Tragedia De Tlahuelilpan. Editorial: Colegio Libre de Hidalgo.

Kim, J., Tol, W. A., Shrestha, A., Kafle, H. M., Rayamajhi, R., Luitel, N. P., ...Thapa, L., \& Surkan, P. J. (2017). Persistent Complex Bereavement Disorder and Culture: Early and Prolonged Grief in Nepali Widows, Psychiatry $\quad$ Spring, http://dx.doi.org/10.1080/00332747.2016.1213560

Kinyuy Lange, R. (2016). Psychological Elements of Trauma and Grief among Young Banso Widows in Cameroon. $\quad$ Oregon University. https://ir.library.oregonstate.edu/concern/graduate_thesis_or_dissertations/qj72pb299

Lambert, J. E., Witting, A. B., Anderson, S., Ponnamperuma, \& L., Wickrama, T. (2017). Posttraumatic Cognitions and Depressive Symptoms in War and Disaster Affected Widows in Sri Lanka: The Role of Community Support. Contemporary Family Therapy. (0), 1-8. https://doi.org/10.1007/s10591-017-9441

López-Pozos, C. (2014). Cicatrices abiertas: lo que el trauma nos dejó. En (Eds.), López-Pozos, C.; Domínguez-Ángel, M.; Aragón-Domínguez, M.; Rosa-Zamora, M.E. \& Xancatl-Flores, F. Organización Social y Calidad de vida (pp.109-134). Universidad Autónoma de Tlaxcala. 
Luna-Nemecio, J., Tobón, S., \& Juárez-Hernández, L. J. (2020). Sustainability-based on socioformation and complex thought or Sustainable Social Development. Resources, $\begin{array}{llll}\text { Environment and Sustainability, } & \text { (2),1-8. } 100007 .\end{array}$ https://doi.org/10.1016/j.resenv.2020.100007

Lundin, T. (1984). Morbidity following Sudden and Unexpected Bereavement. Bristish Journal of Psichiatry, 144(1), 84-88. DOI https://doi.org/10.1192/bjp.144.1.84

Manual Diagnóstico y Estadístico de Trastornos Mentales. (2002). Trastornos del Estado de Ánimo. Barcelona: Editorial Masson.

MansourGhaith, S., SalmanAL-Baddareen, G., MousaAl Ali, T., Mohammed, A. M. (2020). Apoyo social percibido entre las mujeres viudas en Jordania: un estudio exploratorio. Foro Internacional de Estudios de la Mujer, Mayo-Junio s/p DOI: https://doi.org/10.1016/j.wsif.2020.102364

Martín-Baró, I. (1990). Psicología social de la guerra: trauma y terapia. UCA Editores. San Salvador. El Salvador.

Martínez, M. (2007). Ciencia y arte en la metodología cualitativa. México: Trillas

Medina, J. L. (2015). Trauma psíquico. Madrid: Paraninfo.

Molina, H. (25, de agosto 2019). Tomas clandestinas, en niveles del 2018 pese a estrategia vs. Huachicol. El Economista. https://n9.cl/mhwtm

Mukimulhasan, S., Abbott, C. H., Alaka, R., Vankar, G. K., Arun, K., Chetan, P., Rahul, S., Seema, G., Bhaiya, N., \& Prigerson, H. G. (2013). Prolonged Grief Disorder among Indian Women Widowed by Communal Violence Mukimulhasan. Palliative Care \& Medicine Palliat, 3(6), 16. : https://doi.org/10.4172/2165-7386.100016

Omozusi Omosefe, M., Ebere Miracle, K., Oluwafikayomi Opeyemi, B., Olaoye, T., end Okondu Ogechuckwu, E. (2018). Social Supports and Widows in Churches: An Empirical Assessment in Nigeria Context, Journal of Education and Practice, 9(27), 8-15. https://www.iiste.org/Journals/index.php/JEP/article/view/44252/45651

Plan de Desarrollo Municipal, Tlahuelilpan (2016-2020). https://n9.cl/gx2qw

Pragya, K., Basnet, S., \& Prabhat, L. (2018). Depression and anxiety among war-widows of Nepal: a post-civil war cross-sectional study Syaron. Psychology, Health \& Medicine, 23(2), 141-153. https://doi.org/10.1080/13548506.2017.1338735

Rosenblatt, P. C. (2008). Grief across cultures: A review and research agenda. In Stroebe, M. S., Hansson, R. O., Schut, H. y Stroebe, W. (Eds.), Handbook of bereavement research and practice: Advances in theory and intervention. American Psychological Association, 207222. https://doi.org/10.1037/14498-010.

Sharma, N. \& Lehal, R. (2018). A study of psychosocial challenges of army personnel widow "war begins after its end. International Research Journal of Human Resources and Social Sciences, 5(02), February, 74-91. https://1bestlinks.net/8PDB3

Worden, W. (1997). El tratamiento del duelo: asesoramiento psicológico y terapia. Barcelona: Paidós 